\title{
Dengue vector control strategies in an urban setting: an economic modelling assessment
}

\author{
Paula Mendes Luz, Tazio Vanni, Jan Medlock, A David Paltiel, and Alison P Galvani \\ Department of Epidemiology and Public Health, Yale University School of Medicine, New Haven, \\ CT, USA (P M Luz PhD, J Medlock PhD, Prof A D Paltiel PhD, A P Galvani PhD); Evandro \\ Chagas Clinical Research Institute, Oswaldo Cruz Foundation, Rio de Janeiro, Brazil (P M Luz); \\ Department of Public Health and Policy, London School of Hygiene and Tropical Medicine, \\ London, UK (T Vanni MD); and Department of Mathematical Sciences, Clemson University, \\ Clemson, SC, USA (J Medlock)
}

\section{Summary}

Background-An estimated 2.5 billion people are at risk of dengue. Incidence of dengue is especially high in resource-constrained countries, where control relies mainly on insecticides targeted at larval or adult mosquitoes. We did epidemiological and economic assessments of different vector control strategies.

Methods-We developed a dynamic model of dengue transmission that assesses the evolution of insecticide resistance and immunity in the human population, thus allowing for long-term evolutionary and immunological effects of decreased dengue transmission. We measured the dengue health burden in terms of disability-adjusted life-years (DALYs) lost. We did a costeffectiveness analysis of 43 insecticide-based vector control strategies, including strategies targeted at adult and larval stages, at varying efficacies (high-efficacy [90\% mortality], mediumefficacy [60\% mortality], and low-efficacy [30\% mortality]) and yearly application frequencies (one to six applications). To assess the effect of parameter uncertainty on the results, we did a probabilistic sensitivity analysis and a threshold analysis.

Findings-All interventions caused the emergence of insecticide resistance, which, with the loss of herd immunity, will increase the magnitude of future dengue epidemics. In our model, one or more applications of high-efficacy larval control reduced dengue burden for up to 2 years, whereas three or more applications of adult vector control reduced dengue burden for up to 4 years. The incremental cost-effectiveness ratios of the strategies for two high-efficacy adult vector control applications per year was US\$615 per DALY saved and for six high-efficacy adult vector control applications per year was $\$ 1267$ per DALY saved. Sensitivity analysis showed that if the cost of adult control was more than 8.2 times the cost of larval control then all strategies based on adult control became dominated.

Correspondence to: Dr Paula Mendes Luz, Evandro Chagas Clinical Research Institute, Oswaldo Cruz Foundation, Avenida Brasil 4365, Rio de Janeiro 21040-360, Brazil paula.luz@ipec.fiocruz.br.

See Online for webappendix

Contributors

PML had the idea for the study and drafted the paper. PML and TV did the cost-effectiveness analysis and the sensitivity analysis, interpreted the results, and discussed the findings. PML and JM developed the mathematical model. ADP and APG assisted in the design of the study, interpretation of results, and discussion of the findings. All authors revised the paper for important intellectual content. All authors have approved the final version of the paper.

Conflicts of interest

We declare that we have no conflicts of interest. 
Interpretation-Six high-efficacy adult vector control applications per year has a costeffectiveness ratio that will probably meet WHO's standard for a cost-effective or very costeffective intervention. Year-round larval control can be counterproductive, exacerbating epidemics in later years because of evolution of insecticide resistance and loss of herd immunity. We suggest the reassessment of vector control policies that are based on larval control only.

Funding-The Fulbright Programme, CAPES (Brazilian federal agency for post-graduate education), the Miriam Burnett trust, and the Notsew Orm Sands Foundation.

\section{Introduction}

About 50 million dengue infections occur every year worldwide. ${ }^{1}$ The incidence of dengue is increasing, especially in metropolitan areas, mainly because of growing vector and human population densities. Dengue incidence is high in resource-constrained countries where restricted health budgets are divided between control and treatment. Dengue control relies mainly on insecticides targeted at larval or adult mosquitoes. ${ }^{2,3}$ However, the evolution of insecticide resistance can lead to the failure of dengue control programmes. ${ }^{4,5}$

To estimate the effect of insecticide-based vector control on health and health economic outcomes, we developed a dengue transmission model that extends previous analyses of vector control ${ }^{6-8}$ by considering the evolution of insecticide resistance in mosquito populations together with human population immunity, and by integration of a dynamic model with an economic assessment. We use the city of Rio de Janeiro in Brazil as a case study because it is an example of a resource-constrained urban setting with endemic dengue, and because adequate data are available for setting the parameters of the model. We used the model to calculate the health burden in terms of disability-adjusted life years (DALYs) lost to dengue and the cost-effectiveness of various interventions.

\section{Methods}

\section{Mathematical model}

To describe the transmission cycle of dengue in a metropolitan area, we extended a previous mosquito $\operatorname{model}^{9}$ to include human population dynamics and dengue transmission (webappendix p 4). The mosquito model incorporates seasonality and population genetics of insecticide-resistance evolution. The model parameters were set with ecological and biological data specific to Aedes aegypti, retrieved through searches of PubMed, Web of Science, SciELO, reports from the Brazilian government, and WHO publications (webappendix p 5). ${ }^{9}$ To simulate dengue transmission, adult mosquitoes were divided into two groups: uninfected old adult (older than 5 days; $\mathrm{O}$ ), and dengue-virus infected adult mosquitoes $\left(\mathrm{O}_{\mathrm{I}}\right)$. Uninfected old adults acquire infection (entering the compartment $\mathrm{O}_{\mathrm{I}}$ ) at a rate that depends on the force of infection $\left(\lambda_{\mathrm{M}}\right)$ and the duration of the extrinsic incubation period $(\tau)$. We defined the force of infection as a function of biting rate of mosquitoes (c); proportion of people with infection (either primary or secondary infections; I), and their respective probabilities of infecting a mosquito, $\left(\beta_{1} \mathrm{I}_{1}+\beta_{2} \mathrm{I}_{2}\right) / \mathrm{H}$, where $\mathrm{H}$ is the total number of human beings; and mosquitoes' susceptibility to infection $\left(\square_{M}\right)$. Thus, $\lambda_{M}=\left(c \square_{M}\left(\beta_{1} I_{1}+\right.\right.$ $\left.\left.\beta_{2} \mathrm{I}_{2}\right)\right) / \mathrm{H}$.

We incorporated the demographic dynamics of the human population from Rio de Janeiro into the model (webappendix p 5). Newborn babies $\left(R_{m}\right)$ have maternally acquired immunity that wanes at rate $a$. Susceptible individuals $\left(S_{1}\right)$ acquire primary infection at the force of infection rate $\left(\lambda_{1}\right)$, which depends on the biting rate of mosquitoes; the proportion of infected mosquitoes and their infectivity, $\beta_{\mathrm{m}} \mathrm{O}_{\mathrm{I}} / \mathrm{O}_{\mathrm{T}}$, where $\mathrm{O}_{\mathrm{T}}$ is the total number of 
adult mosquitoes; the density of mosquitoes to people, $\mathrm{O}_{\mathrm{T}} / \mathrm{H}$; and the susceptibility of people to primary infection, $\square_{1}$. Thus, $\lambda_{1}=\left(\mathrm{c} \square_{1}\left(\beta_{\mathrm{m}} \mathrm{O}_{\mathrm{I}}\right)\right) / \mathrm{H}$.

The mortality rate for individuals with dengue haemorrhagic fever is given by $\square_{1}$. Most human beings with primary infection $\left(\mathrm{I}_{1}\right)$ recover at rate $(\gamma)$ into a cross-immunity compartment (immunity to all four dengue serotypes; $\mathrm{R}_{1}$ ). Cross-immunity wanes at a rate $\delta$, resulting in susceptibility to secondary infection $\left(\mathrm{S}_{2}\right)$. Secondary infection $\left(I_{2}\right)$ occurs at a rate $\lambda_{2}$. This force of secondary infection depends on the biting rate of mosquitoes; the proportion of infected mosquitoes and their infectivity; the density of mosquitoes to human beings; and the susceptibility of people to secondary infection $\left(\square_{2}\right)$. Thus, $\lambda_{2}=$ $\left(\mathrm{c} \square_{2}\left(\beta_{\mathrm{m}} \mathrm{O}_{\mathrm{I}}\right)\right) / \mathrm{H}$. Most individuals with secondary infection, recover at rate $\gamma$ into a resistant compartment $\left(\mathrm{R}_{2}\right)$. Those presenting with dengue haemorrhagic fever die with probability $\square_{2}$ (which is greater than $\square_{1}$ because secondary infections are more severe). ${ }^{1}$ Thus, as with previous analyses, ${ }^{10}$ we modelled primary and secondary infections, but did not specify the dengue serotype causing the infection. Despite this simplification, we incorporated the force of infection for all four serotypes by defining full susceptibility to primary infection and $75 \%$ susceptibility to secondary infection resulting from immunity to the primary serotype.

\section{Insecticide-based vector control}

We assessed the effect of vector control for a 5-year period. Two forms of vector control were analysed-larval and adult control. Larval control has an environmental persistence of 2 months, ${ }^{11,12}$ during which the effectiveness of the insecticide wanes (webappendix p 3). Adult control with ultra-low-volume insecticide applications has an immediate effect on the mosquito population that lasts for 1 day (webappendix p 3). ${ }^{13,14}$ Larval and adult control interventions consisted of one to six applications every year. Because field-based estimates of vector control efficacy vary substantially as a function of many factors, ${ }^{15,16}$ we explored a full range of efficacies. For both forms of control, we assessed the effect of high-efficacy ( $90 \%$ mortality), medium-efficacy (60\% mortality), and low-efficacy (30\% mortality) interventions. We also assessed six combined strategies: one application of high-efficacy larval control with either three, four, or five applications of low-efficacy adult control, and two applications of high-efficacy larval control with either three, four, or five applications of low-efficacy adult control. Thus, including no vector control, 43 vector control strategies were considered.

\section{Health outcomes and costs}

DALYs were used to measure health outcomes because they incorporate both death and disability. ${ }^{17}$ Details of DALY calculations, including parameter values, are shown in the webappendix ( $\mathrm{p} 7$ ). We considered the costs of larval and adult control in a large metropolitan area (webappendix $\mathrm{p}$ 6). In conformity with international standards for economic assessments, ${ }^{18}$ we did the analysis from a societal perspective, assessing direct medical and non-medical costs and indirect costs from workdays lost because of dengue (webappendix p 6). ${ }^{19,20}$ Costs were expressed in 2009 US dollars (US\$). Costs and DALYs were discounted at a yearly rate of $3 \%$, as per WHO recommendations. ${ }^{21}$

\section{Cost-effectiveness analysis of vector control}

We calculated the incremental cost-effectiveness of all 43 vector control strategies. Comparative value was measured in \$ per DALY saved during the 5-year vector control assessment period. We defined Brazil-specific thresholds of $\$ 24660$ per DALY saved for a cost-effective intervention, and of $\$ 8220$ per DALY saved for a very cost-effective intervention, on the basis of the criteria of the WHO Commission on Macroeconomics and Health. ${ }^{21,22}$ 


\section{Sensitivity analysis}

We did probabilistic sensitivity analyses and threshold analyses to examine the effect of parameter uncertainty on the results (webappendix p 9). In the probabilistic sensitivity analysis we assessed the effect of uncertainty in both the DALY and cost parameters. Gamma distributions were assigned to cost parameters, and beta distributions were assigned to probabilities. By sampling from these distributions, 10000 estimates for the costs and effects of each strategy were calculated. Cost-effectiveness acceptability curves were used to depict the level of uncertainty surrounding our cost-effectiveness estimates at different values of society's willingness to pay for an additional DALY saved. ${ }^{23}$ In view of the uncertainty of available cost estimates for insecticide-based vector control, we did a specific threshold analysis of cost parameters. In the threshold analysis, we established the cost of adult mosquito control beyond which any strategy involving such control would become dominated (a strategy is said to be dominated if it costs more and delivers fewer DALYs than another strategy [strong dominance] or a combination of other strategies [weak dominance]) according to WHO's standard for a cost-effective intervention for Brazil. ${ }^{21,22}$ Statistical analyses were done with MatLab Version 7.5, Microsoft Office Excel 2003, and PopTools Version 3.2.3.

\section{Role of the funding sources}

The sponsors of the study had no role in study design, data collection, data analysis, data interpretation, or writing of the report. The corresponding author had full access to all the data in the study and had final responsibility for the decision to submit for publication.

\section{Results}

Without any vector control intervention, large epidemics of mostly primary infections are predicted to occur over 5 years, with interepidemic periods of lower dengue incidence (figure 1). After this initial period, stable equilibrium is reached because of the deterministic nature of the model, and dengue becomes endemic. Over 20 years, the average yearly incidence of dengue fever is 100 cases per 10000 population, which is consistent with epidemiological data from Brazil and Latin America. ${ }^{24-27}$ Our baseline period of analysis was a 5-year period after dengue established itself endemically (figure 1). For this period, the average annual dengue burden was 227 DALYs lost per million population, which accords with estimates based on empirical data. ${ }^{20,28-32}$ For the entire 5-year period, the expected dengue burden was 1133 DALYs lost per million populations (table).

High-efficacy larval control reduced the dengue health burden during the first 2 years (table; figure 2) - three or more applications of high-efficacy larval control reduced the expected annual dengue burden to below 14 DALYs lost per million population. For all high-efficacy larval control interventions, use of three applications led to the smallest loss of DALYs in the third year, use of one application led to the smallest loss of DALYs in the fourth year, and use of four applications led to the smallest loss of DALYs in the fifth year (table; figure 2). Medium-efficacy larval control resulted in 18 or fewer DALYs lost per million population annually during the first 2 years when six applications of larval control were applied (table; figure 2). However, this same strategy led to the loss of most DALYs (among all intermediate-efficacy larval control interventions) in years 3 and 4. Low-efficacy larval control had little effect on dengue health burden (table; figure 2). For the entire 5-year period, three applications of high-efficacy larval control every year reduced the dengue burden the most (table).

Four or more applications of high-efficacy adult control decreased the number of DALYs lost in the first 4 years (table; figure 3 ). In the fifth year, the most effective high-efficacy 
intervention strategy was two applications of high-efficacy adult vector control. Although use of six applications of medium-efficacy adult control was the most effective mediumefficacy strategy in the first 4 years, it was the least effective strategy in the fifth year (table; figure 3). Low-efficacy adult vector control offered sustained reduction of the dengue health burden in the first 2 years when used at a frequency of six applications (table; figure 3 ). For the entire 5-year period, six applications of high-efficacy adult vector control reduced the dengue burden to the greatest extent, resulting in 248 DALYs lost per million individuals.

Of the combined intervention strategies, those with two applications of high-efficacy larval control substantially reduced dengue burden in the first 3 years. However, a severe epidemic occurred in year 4 (table; figure 4). If only one high-efficacy larval control application was used in combination with low-efficacy adult control, epidemics were predicted to occur in the third and fourth years, but the magnitude of these epidemics was lower than that of the epidemic expected in the fourth year with use of two high-efficacy larval control applications. During the 5-year period, one high-efficacy larval control application and five low-efficacy adult control applications reduced the dengue burden to the greatest extent (table). Of all vector control strategies, the strategy that most substantially reduced the number of DALYs lost per million population during the 5-year period was six applications of high-efficacy adult vector control (table).

Figure 5 shows all 43 interventions in the cost-effectiveness plane, which depicts the difference in costs and effectiveness between the strategies. Cost-effectiveness analysis showed that three strategies were non-dominated: no control, use of two applications of high-efficacy adult control, and use of six applications of high-efficacy adult control (figure 5). One and five applications of high-efficacy adult control were weakly dominated strategies. All other strategies were dominated. ${ }^{33}$ The incremental cost-effectiveness ratio for two high-efficacy adult control applications was $\$ 615$ per DALY saved and for six highefficacy adult control applications was $\$ 1267$ per DALY saved.

The probabilistic sensitivity analysis (figure 6) showed that use of six applications of adult control would meet WHO's standard for cost effective with probabil ity $99 \%$ and the standard for very cost effective with probability $89 \%$. Threshold analysis showed that if the cost of adult control was more than $8 \cdot 2$ times the cost of larval control then all strategies involving adult control became dominated.

\section{Discussion}

In our model, one or more applications of high-efficacy larval control reduced dengue burden for up to 2 years, whereas three or more applications of high-efficacy adult vector control reduced dengue burden for up to 4 years. The WHO Commission on Macroeconomics and Health suggests that interventions be deemed cost effective in a given country if their cost-effectiveness ratio is less than three times the national per head gross domestic product. With use of this standard, we showed that the intervention consisting of six high-efficacy adult control applications is cost effective over a wide range of epidemiologically realistic parameter values.

Year-round larval control is used in many settings where dengue transmission occurs. ${ }^{34,35}$ However, our analyses indicate that strategies based on continuous larval control could be counterproductive. Initially, such strategies reduce the mosquito population and suppress dengue transmission. Indeed, our results show that only a few DALYs are lost per million population during the first 2 years. However, as insecticide resistance evolves and, consequently, the effect of vector control decreases, the mosquito population recovers. Additionally, the suppressed dengue transmission during the initial years of control results in 
a loss of herd immunity in the human population. Growth of the insecticide-resistant mosquito populations coupled with the loss of herd immunity increases the magnitude of the eventual epidemic when the DALYs lost per million population offsets the predicted dengue burden.

Our model extends previous analyses ${ }^{6-8}$ by considering the evolution of insecticide resistance together with the loss of herd immunity, thus accounting for the long-term effects of reductions in dengue transmission. Our predictions of a rapid evolution of insecticide resistance in the mosquito population accord with data from other studies. ${ }^{36}$ In Rio de Janeiro, for example, a substantial rise in the prevalence of resistance was recorded in a 2year period. ${ }^{4}$ Furthermore, we linked the dynamic model with a health burden analysis and a cost-effectiveness analysis. The probabilistic sensitivity analysis allowed us to assess the probability of a strategy being cost effective with parameter uncertainty and willingness-topay uncertainty. Using a threshold analysis, we were able to show the extent to which the cost of adult vector control would have to offset the cost of larval control for adult vector control strategies to become dominated.

The results of our uncertainty analysis are presented with use of multiway cost-effectiveness acceptability curves as suggested by a broad consensus of experts. ${ }^{37,38}$ Concern has been raised that cost-effectiveness acceptability curves can violate the economic assumption of independence of irrelevant alternatives and that pairwise comparisons might be more appropriate. ${ }^{39}$ This discrepancy can arise if one or more of the strategies under consideration is politically or administratively infeasible, or if the analysis is directed at different decision makers, not all of whom agree on the set of viable choices. In either situation, inclusion of irrelevant strategies in such a comparison would negatively affect the assessment of other, more viable options. In our analysis, however, none of the options were politically or administratively infeasible, nor was our analysis aimed at decision makers with differing opinions on the set of viable choices. Pairwise comparisons would not account for the interaction between all strategic choices avail able to decision makers and would therefore be uninterpretable.

A limitation of our economic assessment is that it was done in the context of an urban area with endemic dengue. Thus, our results are generalisable to settings with endemic dengue, where epidemics of fairly constant size are expected. In settings where dengue transmission dynamics are erratic, with introduction, elimination, and reintroduction of serotypes, our results could be less applicable because epidemic size can vary by year.

Ideally, our analysis would have used local cost estimates. However, vector control spending data for Brazil were not available. Instead, we obtained cost parameter estimates from data from other countries (webappendix p 6). In recognition of the uncertainty surrounding these parameters, we did extensive probabilistic sensitivity analysis and threshold analysis.

Although our cost-effectiveness results were robust to uncertainty in the cost parameters, our findings should be interpreted with caution. We recommend studies of vector control resource use as an important area for further investigation that will better inform future costeffectiveness analyses.

In the present study, costs and effectiveness were assessed for a 5-year period. Guidelines on cost-effectiveness analysis recommend the use of a period appropriate for the assessments of all disease-specific costs, and of costs and effectiveness of the interventions being assessed. ${ }^{40}$ The effect of vector control could extend beyond our 5-year period. However, interactive factors related to the human population (demography and immunity), vectors (fitness and insecticide resistance), vector and human population dynamics (relative population sizes and patterns of urbanisation), and the possible development of other 
technologies for disease treatment and prevention (such as a vaccine) introduce substantial uncertainty when modelling for longer periods, suggesting that the 5-year period used in this study is justified as an initial assessment.

More studies of insecticide delivery, efficacy, and effect are urgently needed. Most studies that assessed field-based efficacy of adult and larval control were done more than two decades ago. ${ }^{15,16}$ The theoretical efficacy of insecticides measured under laboratory conditions is quite different from what is achieved when applied in real conditions. Such studies would be important for the guidance of future economic analyses of vector control.

Although our analysis was based mainly on data from metropolitan areas of Brazil, the results are likely to be applicable to other urban settings with endemic dengue. Our model incorporates location-specific data for climate (temperature and rainfall), human population size and demographics, and, whenever possible, locally estimated biological model parameters for the mosquito population. These parameters could be adjusted to represent other locations. Moreover, the model could be extended to incorporate other available interventions such as breeding site elimination ${ }^{41}$ or other vector control interventions such as insecticide-treated curtains or water container covers ${ }^{42}$ in addition to potential new interventions, such as vaccines ${ }^{43}$ and release of genetically modified mosquitoes. ${ }^{44,45}$ Indeed, if included in the model, these factors could affect our findings. We encourage similar economic assessments for settings with endemic dengue to better understand the best practices of dengue control and how they vary according to location-specific factors. The optimum use of the model developed in this study would use up-to-date data from mosquito and dengue case surveillance systems, which would allow for adaptable strategies to be constructed as dengue dynamics unfold in a given place and time.

Dengue control is a challenging endeavour, especially because $A$ aegypti evolves in response to control interventions (ie, development of insecticide resistance), because herd immunity is affected by reduced transmission associated with control, and because viral reintroduction can occur from other locations, making dengue elimination unlikely. Our model shows that when the effect of insecticide-based interventions wane because of the evolution of insecticide resistance, pronounced epidemics will occur, the severity of which depends on the specific intervention used. These findings draw attention to the importance of considering insecticide resistance and dengue transmission dynamics when implementing insecticide-based dengue vector control programmes in a specific setting.

\section{Supplementary Material}

Refer to Web version on PubMed Central for supplementary material.

\section{Acknowledgments}

PML was funded by The Fulbright Programme, CAPES (Brazilian federal agency for post-graduate education), and the Miriam Burnett trust. APG was funded by Notsew Orm Sands Foundation and the Miriam Burnett trust. JM was supported by the National Science Foundation (grant SBE-0624117) and the Notsew Orm Sands Foundation. We gratefully acknowledge support from NIH MIDAS (grant UO1 GM087719-01).

\section{References}

1. Guzman MG, Kouri G. Dengue: an update. Lancet Infect Dis. 2002; 2:33-42. [PubMed: 11892494]

2. Gubler DJ. Epidemic dengue/dengue hemorrhagic fever as a public health, social and economic problem in the 21st century. Trends Microbiol. 2002; 10:100-03. [PubMed: 11827812] 
3. Hemingway J, Beaty BJ, Rowland M, Scott TW, Sharp BL. The innovative vector control consortium: improved control of mosquito-borne diseases. Trends Parasitol. 2006; 22:308-12. [PubMed: 16713358]

4. Cunha MP, Lima JB, Brogdon WG, Moya GE, Valle D. Monitoring of resistance to the pyrethroid cypermethrin in Brazilian Aedes aegypti (Diptera: Culicidae) populations collected between 2001 and 2003. Mem Inst Oswaldo Cruz. 2005; 100:441-44. [PubMed: 16113895]

5. Gratz NG, Jany WC. What role for insecticides in vector control programs? Am J Trop Med Hyg. 1994; 50:11-20. [PubMed: 8024077]

6. Burattini MN, Chen M, Chow A, et al. Modelling the control strategies against dengue in Singapore. Epidemiol Infect. 2008; 136:309-19. [PubMed: 17540051]

7. Focks DA, Daniels E, Haile DG, Keesling JE. A simulation-model of the epidemiology of urban dengue fever-literature analysis, model development, preliminary validation, and samples of simulation results. Am J Trop Med Hyg. 1995; 53:489-506. [PubMed: 7485707]

8. Newton EA, Reiter P. A model of the transmission of dengue fever with an evaluation of the impact of ultra-low volume (ULV) insecticide applications on dengue epidemics. Am J Trop Med Hyg. 1992; 47:709-20. [PubMed: 1361721]

9. Luz PM, Codeco CT, Medlock J, Struchiner CJ, Valle D, Galvani AP. Impact of insecticide interventions on the abundance and resistance profile of Aedes aegypti. Epidemiol Infect. 2009; 137:1203-15. [PubMed: 19134235]

10. Bartley LM, Donnelly CA, Garnett GP. The seasonal pattern of dengue in endemic areas: mathematical models of mechanisms. Trans R Soc Trop Med Hyg. 2002; 96:387-97. [PubMed: 12497975]

11. Lima JB, Melo NV, Valle D. Persistence of Vectobac WDG and Metoprag S-2G against Aedes aegypti larvae using a semi-field bioassay in Rio de Janeiro, Brazil. Rev Inst Med Trop Sao Paulo. 2005; 47:7-12. [PubMed: 15729468]

12. Pontes RJ, Regazzi AC, Lima JW, Kerr-Pontes LR. Residual effect of commercial applications of larvicides temefos and Bacillus thuringiensis israelensis on Aedes aegypti larvae in recipients with water renewal. Rev Inst Med Trop Sao Paulo. 2005; 38:316-21.

13. Koenraadt CJ, Aldstadt J, Kijchalao U, Kengluecha A, Jones JW, Scott TW. Spatial and temporal patterns in the recovery of Aedes aegypti (Diptera: Culicidae) populations after insecticide treatment. J Med Entomol. 2007; 44:65-71. [PubMed: 17294922]

14. Perich MJ, Davila G, Turner A, Garcia A, Nelson M. Behavior of resting Aedes aegypti (Culicidae: Diptera) and its relation to ultra-low volume adulticide efficacy in Panama City, Panama. J Med Entomol. 2000; 37:541-46. [PubMed: 10916294]

15. Gratz NG. Emergency control of Aedes aegypti as a disease vector in urban areas. J Am Mosq Control Assoc. 1991; 7:353-65. [PubMed: 1791444]

16. Gubler DJ. Aedes aegypti and Aedes aegypti-borne disease control in the 1990s: top down or bottom up. Charles Franklin Craig Lecture. Am J Trop Med Hyg. 1989; 40:571-78. [PubMed: 2472746]

17. Murray CJ. Quantifying the burden of disease: the technical basis for disability-adjusted life years. Bull World Health Organ. 1994; 72:429-45. [PubMed: 8062401]

18. Drummond, MF.; Sculpher, MJ.; Torrance, GW.; O'Brien, BJ.; Stoddart, GL. Methods for the economic evaluation of health care programmes. Oxford University Press; Oxford; New York: 2005.

19. McConnell KJ, Gubler DJ. Guidelines on the cost-effectiveness of larval control programs to reduce dengue transmission in Puerto Rico. Rev Panam Salud Publica. 2003; 14:9-16. [PubMed: 12952602]

20. Suaya JA, Shepard DS, Chang MS, et al. Cost-effectiveness of annual targeted larviciding campaigns in Cambodia against the dengue vector Aedes aegypti. Trop Med Int Health. 2007; 12:1026-36. [PubMed: 17875014]

21. WHO. Report of the Commission on Macroeconomics and Health. Geneva: 2001. Macroeconomics and health: investing in health for economic development..

22. International Monetary Fund. [April 3, 2011] Data and statistics. http://www.imf.org/external/data.htm 
23. Briggs, A.; Claxton, K.; Sculpher, M. Decision Modelling for Health Economic Evaluation. Oxford University Press; 2007.

24. de Simone TS, Nogueira RM, Araujo ES, et al. Dengue virus surveillance: the co-circulation of DENV-1, DENV-2 and DENV-3 in the State of Rio de Janeiro, Brazil. Trans R Soc Trop Med Hyg. 2004; 98:553-62. [PubMed: 15251405]

25. Balmaseda A, Hammond SN, Tellez Y, et al. High seroprevalence of antibodies against dengue virus in a prospective study of schoolchildren in Managua, Nicaragua. Trop Med Int Health. 2006; 11:935-42. [PubMed: 16772016]

26. Teixeira MG, Costa MCN, Guerra Z, Barreto ML. Dengue in Brazil: situation 2001 and trends. Dengue Bull. 2002; 26:70-76.

27. Cordeiro MT, Silva AM, Brito CA, et al. Characterization of a dengue patient cohort in Recife, Brazil. Am J Trop Med Hyg. 2007; 77:1128-34. [PubMed: 18165535]

28. Meltzer MI, Rigau-Perez JG, Clark GG, Reiter P, Gubler DJ. Using disability-adjusted life years to assess the economic impact of dengue in Puerto Rico: 1984-1994. Am J Trop Med Hyg. 1998; 59:265-71. [PubMed: 9715944]

29. Clark DV, Mammen MP, Nisalak A, Puthimethee V, Endy TP. Economic impact of dengue fever/ dengue hemorrhagic fever in Thailand at the family and population levels. Am J Trop Med Hyg. 2005; 72:786-91. [PubMed: 15964964]

30. Armien B, Suaya JA, Quiroz E, et al. Clinical characteristics and national economic cost of the 2005 dengue epidemic in Panama. Am J Trop Med Hyg. 2008; 79:364-71. [PubMed: 18784227]

31. Garg P, Nagpal J, Khairnar P, Seneviratne SL. Economic burden of dengue infections in India. Trans R Soc Trop Med Hyg. 2008; 102:570-77. [PubMed: 18402995]

32. Luz PM, Grinsztejn B, Galvani AP. Disability adjusted life years lost to dengue in Brazil. Trop Med Int Health. 2009; 14:237-46. [PubMed: 19171013]

33. Hunink, MGM. Decision making in health and medicine: integrating evidence and values. Cambridge University Press; New York: 2001.

34. Bangs MJ, Larasati RP, Corwin AL, Wuryadi S. Climatic factors associated with epidemic dengue in Palembang, Indonesia: implications of short-term meteorological events on virus transmission. Southeast Asian J Trop Med Public Health. 2006; 37:1103-16. [PubMed: 17333762]

35. Donalisio MR, Glasser CM. Entomological surveillance and control of dengue fever vectors. Revista Brasileira de Epidemiologia. 2002; 5:259-72.

36. Montella IR, Martins AJ, Viana-Medeiros PF, Lima JB, Braga IA, Valle D. Insecticide resistance mechanisms of Brazilian Aedes aegypti populations from 2001 to 2004. Am J Trop Med Hyg. 2007; 77:467-77. [PubMed: 17827362]

37. Briggs, A. Handling uncertainty in economic evaluation and presenting results.. In: Drummond, MF.; McGuire, A., editors. Economic evaluation in health care. Oxford University Press; Oxford: 2001. p. 172-241.

38. Fenwick, E. Acceptability curves and confidence ellipses.. In: Kattan, MW., editor. Encyclopedia of medical decision making. Sage Publications; California: 2009. p. 1-7.

39. Jakubczyk M, Kaminski B. Cost-effectiveness acceptability curves-caveats quantified. Health Econ. 2010; 19:955-63. [PubMed: 19688780]

40. Gold, MR. Cost-effectiveness in health and medicine. Oxford University Press; New York: 1996.

41. Fernandez EA, Leontsini E, Sherman C, et al. Trial of a community-based intervention to decrease infestation of Aedes aegypti mosquitoes in cement washbasins in El Progreso, Honduras. Acta Tropica. 1998; 70:171-83. [PubMed: 9698263]

42. Kroeger A, Lenhart A, Ochoa M, et al. Effective control of dengue vectors with curtains and water container covers treated with insecticide in Mexico and Venezuela: cluster randomised trials. BMJ. 2006; 332:1247-52. [PubMed: 16735334]

43. Shepard DS, Suaya JA, Halstead SB, et al. Cost-effectiveness of a pediatric dengue vaccine. Vaccine. 2004; 22:1275-80. [PubMed: 15003657]

44. Medlock J, Luz PM, Struchiner CJ, Galvani AP. The impact of transgenic mosquitoes on dengue virulence to humans and mosquitoes. Am Nat. 2009; 174:565-77. [PubMed: 19737112] 
45. Moreira LA, Iturbe-Ormaetxe I, Jeffery JA, et al. A Wolbachia symbiont in Aedes aegypti limits infection with dengue, Chikungunya, and Plasmodium. Cell. 2009; 139:1268-78. [PubMed: 20064373] 


\section{Panel: Research in context}

Systematic review

PubMed, Web of Science, and SciELO with the search terms "dengue", "model", "aedes aegypti", and "vector" for dengue modelling studies, giving priority to studies from Brazil, then studies done in other parts of the Americas, then studies from other settings. We also searched reports from the Brazilian Government and WHO publications.

Interpretation

Previous modelling studies have shown that insecticide-based larval and adult vector control strategies can reduce dengue incidence. Whereas previopus models take into account transmission dynamics and the effects of seasonality, ours is the first study to assess the effect of vector control in the presence of evolution of insecticide resistance in mosquito populations. Furthermore, we integrate a dynamic model with an economic assessment. Our study draws attention to the importance of the acknowledgment of both the evolution of insecticide resistance in mosquito populations and of herd immunity in the human population when considering insecticide-based vector control strategies for dengue. 


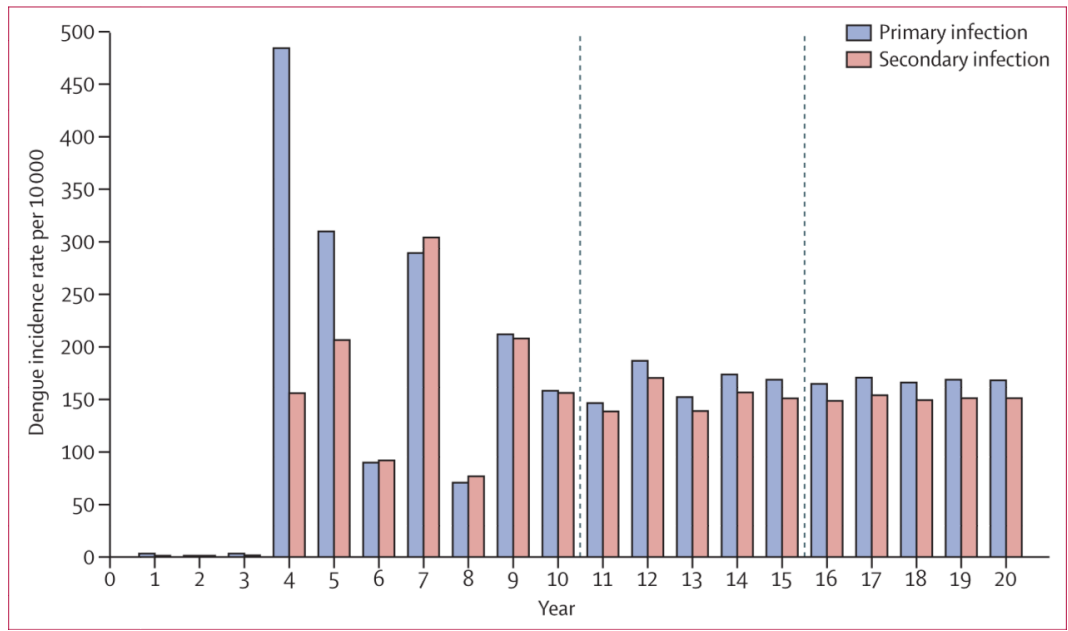

Figure 1. Model of predicted incidence of dengue with no vector control

The area between the dashed lines shows the 5-year vector control assessment window. 


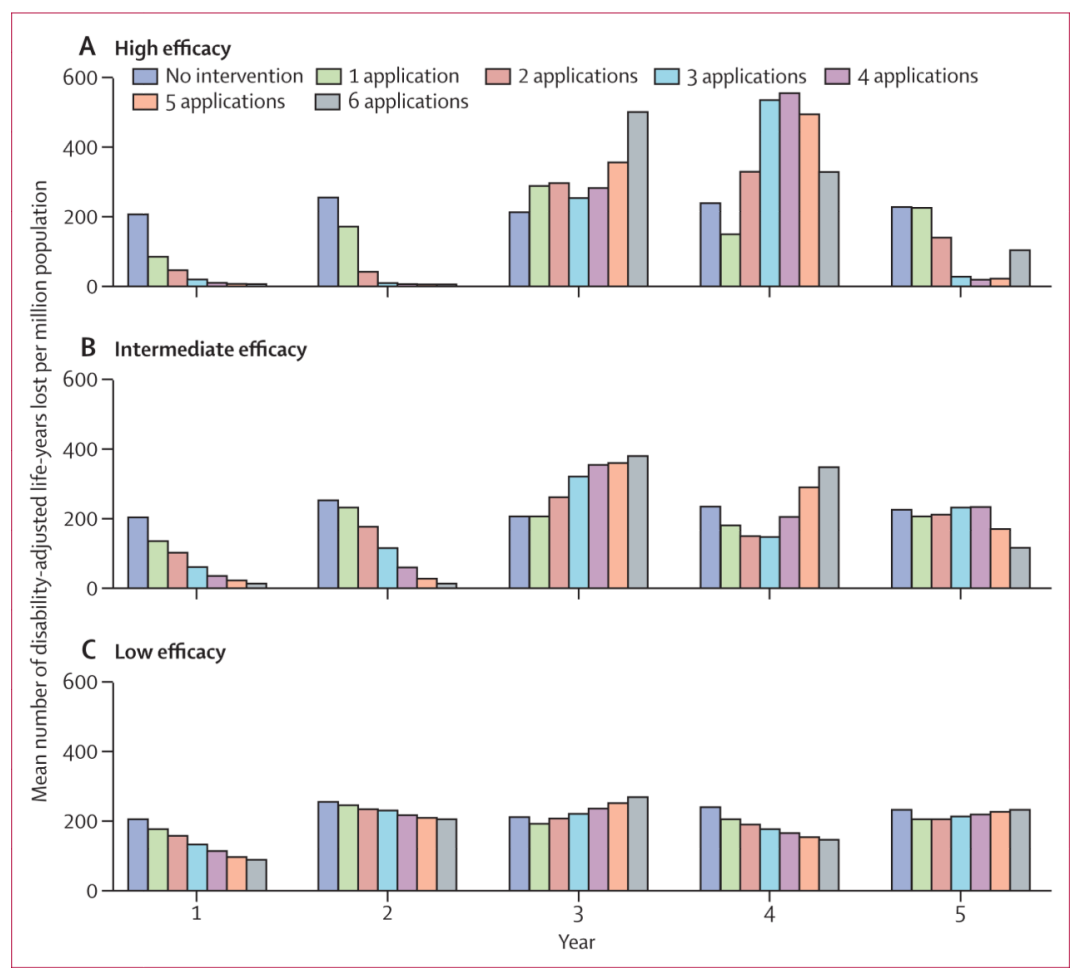

Figure 2.

Effect of larval control on dengue burden 


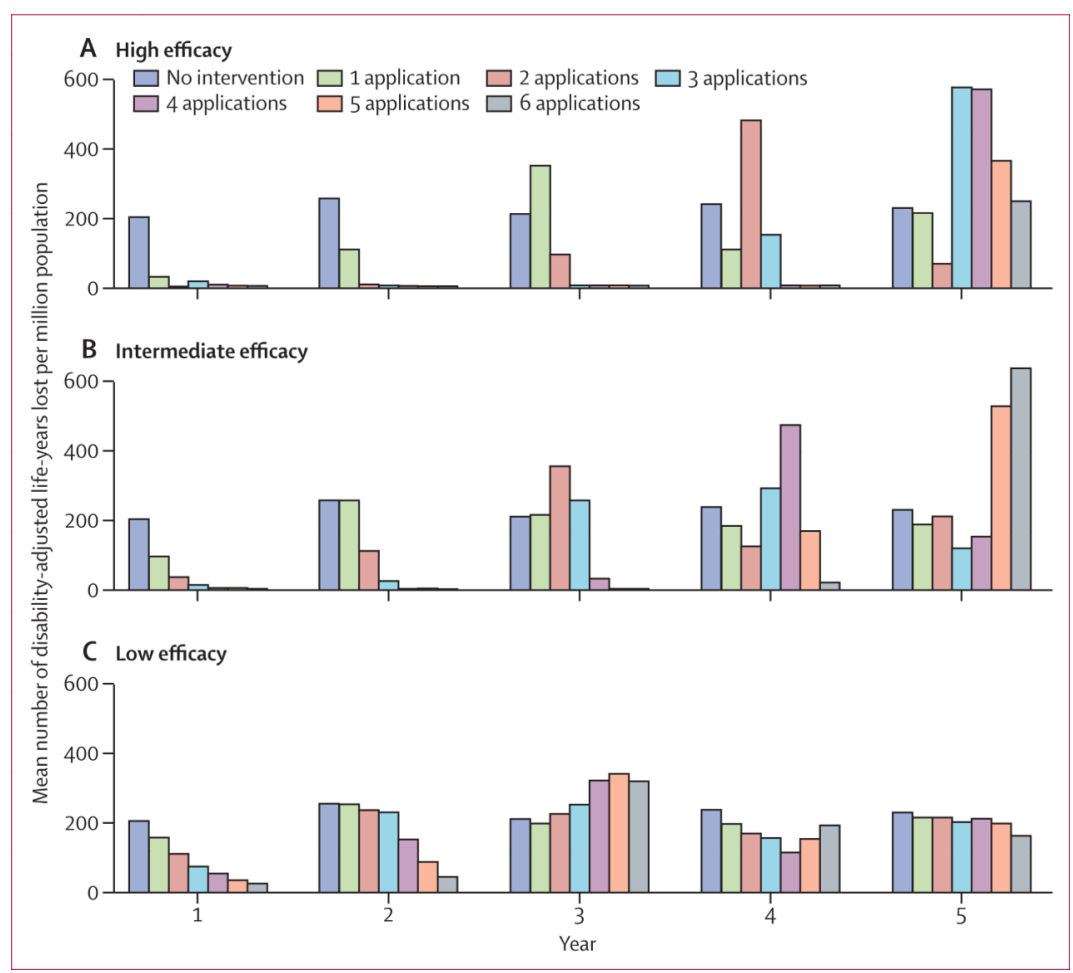

Figure 3.

Effect of adult vector control on dengue burden 


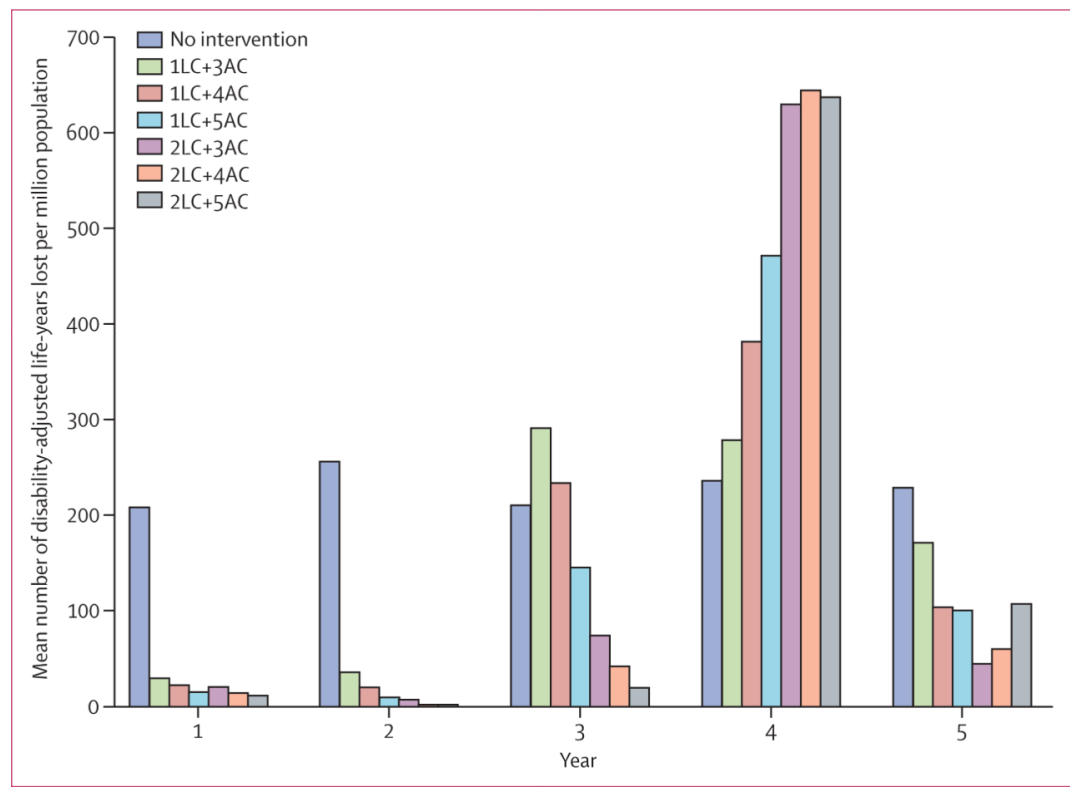

Figure 4. Effect of combined vector control on dengue burden $\mathrm{LC}=$ larval control. AC=adult control. Adjacent numbers refer to numbers of applications. 


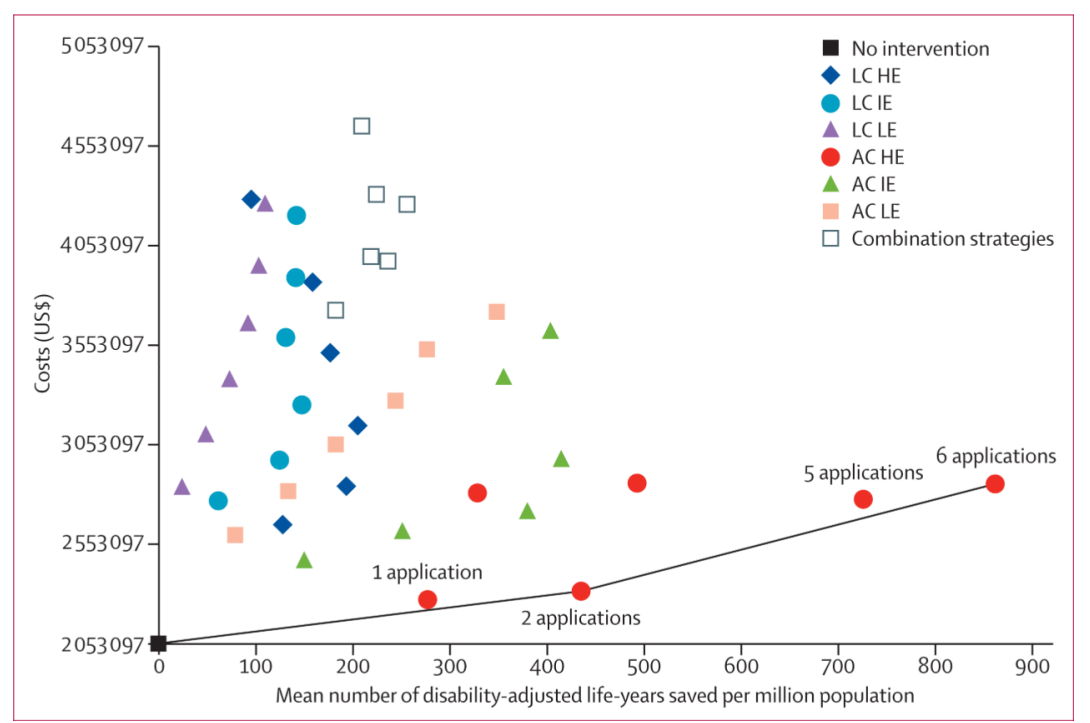

Figure 5. Cost-effectiveness of different control strategies

$\mathrm{LC}=$ larval control. AC=adult control. $\mathrm{HE}=$ high efficacy. IE=intermediate efficacy. LE=low efficacy. The slopes of the black lines represent the incremental cost-effectiveness ratios of the non-dominated strategies. 


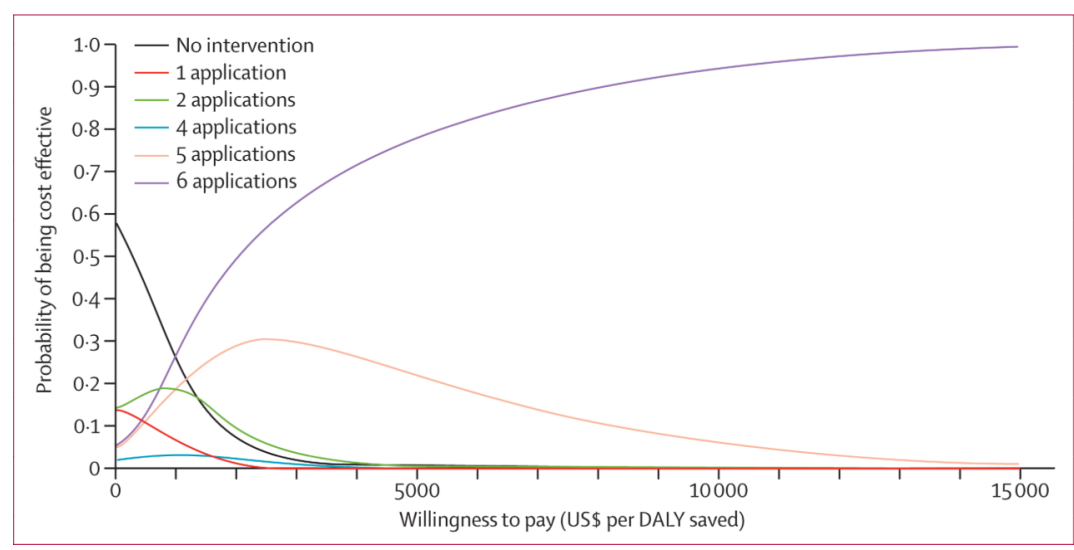

Figure 6. Acceptability curves for control strategies for which the probability of being cost effective is greater than zero

Acceptability curves show the probability that a given intervention meets the WHO definition of cost-effective. 


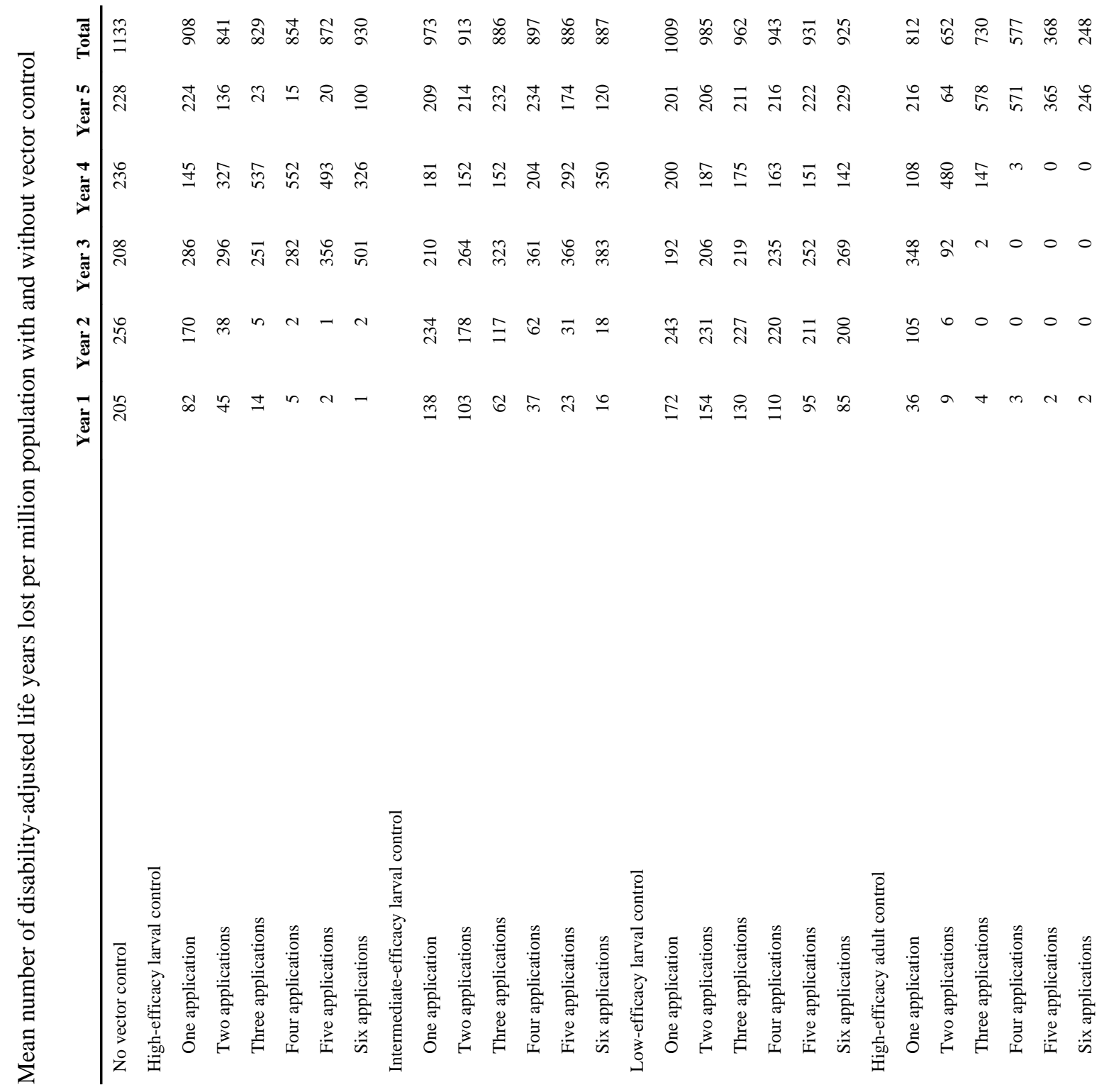




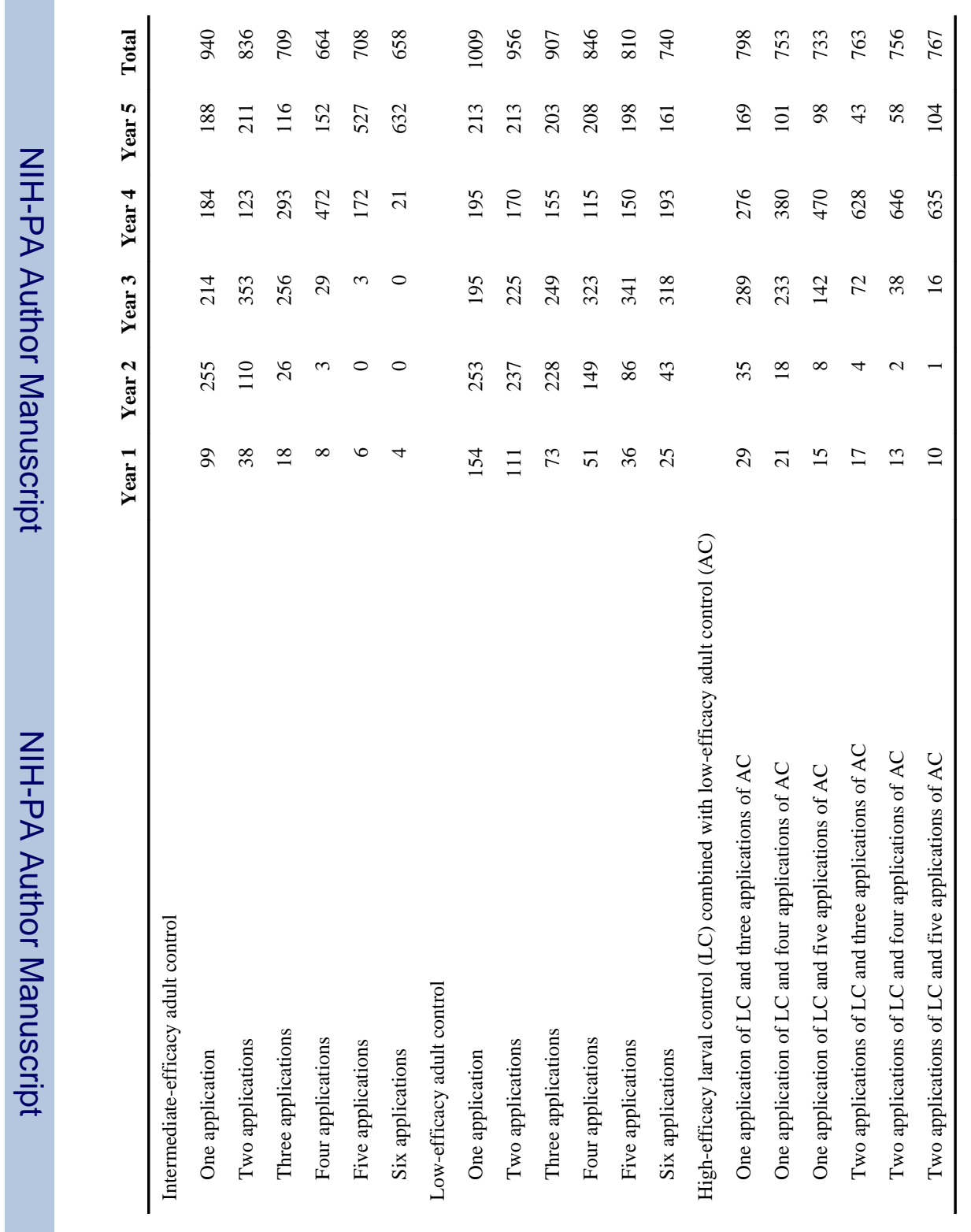

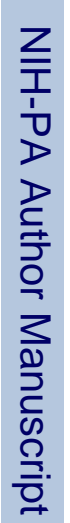

